\title{
SELECTION PRINCIPLES IN THE LAVER, MILLER, AND SACKS MODELS
}

\author{
LYUBOMYR ZDOMSKYY
}

\begin{abstract}
This article is devoted to the interplay between forcing with fusion and combinatorial covering properties. We discuss known instances of this interplay as well as present a new one, namely that in the Laver model for the consistency of the Borel's conjecture, the Hurewicz property is preserved by finite products of metrizable spaces.
\end{abstract}

\section{INTRODUCTION}

Combinatorial covering properties (or selection principles) arguably arose from the study of special sets of reals. These resolved many classical questions in general topology and measure theory. As a result, information about special sets of reals is included in standard topology textbooks, such as Kuratowski's Topology [28]. The most influential survey on special sets of reals is, probably, Miller's chapter 35 in the Handbook of Set-Theoretic Topology. The most recent monograph on this topic is written by Bukovsky, see [14. It complements nicely the classical book [7] of Bartoszynski and Judah. This theory still finds interesting applications in general topology, see, e.g., [23] for the interplay between $\lambda$-sets and homogeneity.

A typical example of the evolution of special sets of reals into selection principles comes from strong measure zero sets (SMZ sets in what follows): $X \subset \mathbb{R}$ is SMZ if for every sequence $\left\langle\epsilon_{n}: n \in \omega\right\rangle$ of positive reals there exists a sequence $\left\langle a_{n}: n \in \omega\right\rangle$ of "centers" such that $X \subset \bigcup_{n \in \omega} B\left(a_{n}, \epsilon_{n}\right)$, where $B(a, \epsilon)=\{x \in \mathbb{R}:|x-a|<\epsilon\}$. SMZ sets were introduced by Borel ca. a century ago who conjectured [13] that only countable sets have such property, i.e., that there are only trivial examples of SMZ sets. This conjecture has been consistently refuted by Sierpiński [51] in 1928, but the question whether Borel's conjecture (BC in what follows) is consistent was answered only in 1976 by Laver in his seminal paper [29]. This outstanding result was the first 1 instance when a forcing, adding a real, was iterated with countable supports without collapsing cardinals. This work of Laver can be thought of as a "birth" of fusion, the latter being a kind of a gentle weakening of the countable completeness which allows to add new reals and

2010 Mathematics Subject Classification. Primary: 03E35, 54D20. Secondary: 54C50, $03 \mathrm{E} 05$.

Key words and phrases. Menger space, Hurewicz space, Laver forcing, Miller forcing, Sacks forcing, fusion.

The author would like to thank the Austrian Science Fund FWF (Grants I 2374-N35 I 3709-N35) for generous support of this research.

${ }^{1}$ According to our colleagues who worked in set theory already in the $70 \mathrm{~s}$. 
nonetheless "treat" countably many dense sets with a single condition, and thus it is also one of the motivations behind Baumgartner's axiom $A$ and Shelah's theory of proper forcing.

In 1938 Rothberger worked on the question whether having SMZ is a topological property, see [43, and introduced the following topological counterpart of SMZ sets which is now known as Rothberger's property and is one of the most intensively studied covering properties: For every sequence $\left\langle\mathcal{U}_{n}: n \in \omega\right\rangle$ of open covers of $X$ there exists a sequence $\left\langle U_{n}: n \in \omega\right\rangle$ such that $U_{n} \in \mathcal{U}_{n}$ for all $n$ and the collection $\left\{U_{n}: n \in \omega\right\}$ is a cover of $X$. Three years later he has shown in 44 that $\mathfrak{b}=\omega_{1}$ implies the existence of a SMZ set which is not Rothberger and hence that having SMZ is not a topological property. Recall that $\mathfrak{b}$ is the minimal cardinality of a subset of $\omega^{\omega}$ which cannot be covered by a $\sigma$-compact one. In the same paper he shows that the $\mathrm{BC}$ is equivalent to all Rothberger metrizable spaces being countable. The Rothberger property is an example of strong combinatorial covering properties in the sense that one selects a single element of each cover, aiming at a "diagonalizing" cover of certain kind. These proved to be important in the study of local properties of spaces of continuous functions, see [2] and references therein.

Another source of selection principles was the dimension theory, where the following property takes its origin: A topological space $X$ has the Menger property (or, alternatively, is a Menger space) if for every sequence $\left\langle\mathcal{U}_{n}: n \in \omega\right\rangle$ of open covers of $X$ there exists a sequence $\left\langle\mathcal{V}_{n}: n \in \omega\right\rangle$ such that each $\mathcal{V}_{n}$ is a finite subfamily of $\mathcal{U}_{n}$ and the collection $\left\{\cup \mathcal{V}_{n}: n \in \omega\right\}$ is a cover of $X$. This property was introduced by Hurewicz, and the current name (the Menger property) is used because Hurewicz proved in [24] that for metrizable spaces his property is equivalent to one property of a base considered by Menger in 31. If in the definition above we additionally require that $\left\{\cup \mathcal{V}_{n}: n \in \omega\right\}$ is a $\gamma$-cover of $X$ (this means that the set $\left\{n \in \omega: x \notin \cup \mathcal{V}_{n}\right\}$ is finite for each $x \in X$ ), then we obtain the definition of the Hurewicz property introduced in [25]. Each $\sigma$-compact space is obviously a Hurewicz space, and Hurewicz spaces have the Menger property. Contrary to a conjecture of Hurewicz the class of metrizable spaces having the Hurewicz property appeared to be much wider than the class of $\sigma$-compact spaces [26, Theorem 5.1]. Moreover, there always exists a subspace of $\omega^{\omega}$ of cardinality $\mathfrak{b}$ all of whose finite powers are Hurewicz, see [8, 9]. Thus we can say that there are always non-trivial examples of metrizable Hurewicz spaces, as contrasted with the behaviour of Rothberger spaces in the Laver model. The properties of Menger and Hurewicz are classical examples of weaker combinatorial covering properties, i.e., properties obtained by selecting finitely many elements from each cover in a given sequence.

The Rothberger property and SMZ sets in the measure theory as well as properties of Menger and Hurewicz in the dimension theory, respectively, are not among the most intensively investigated ones in these areas of mathematics. The reason is that their study required methods developed decades after their introduction. Namely, one of the most fascinating features of these properties as well as many other selection principles is their behavior 
in various models of set theory, which makes forcing and cardinal characteristics one of the main tools in the area. This became apparent from at least the publication of [29], in [6, 16, 17, 36, 50] one can find more recent works along these lines. In what follows we discuss the affect of three classical forcing notions, namely Laver, Miller, and Sacks ones, on selection principles. The unifying theme of all of them is fusion based on the fact that the conditions are certain trees on the natural numbers.

The next after [29] application of the Laver forcing to combinatorial covering properties seems to be [18] where it is proved that in the Laver model, the properties $\alpha_{1}$ and $\alpha_{2}$ introduced by Arkhangel'skil in [1] coincide. Recall that a space $X$ satisfies $\alpha_{2}$ (resp. $\alpha_{1}$ ) if for every countable family $\mathcal{S}$ of non-trivial sequences converging to a point $x \in X$ there exists a sequence $T$ converging to $x$ such that $|T \cap S|=\omega$ (resp. $S \subset^{*} T$ ) for all $S \in \mathcal{S}$. How is this related to covering properties? It has been proved in [58 that for a metrizable space $X$, property $\alpha_{1}$ of $C_{p}(X)$, the space of all continuous functions $f: X \rightarrow \mathbb{R}$ with the topology inherited from the Tychonoff product $\mathbb{R}^{X}$, is equivalent to $X$ having the Hurewicz property with respect to all countable Borel covers. Due to the fact that (unlike the open sets) Borel sets are closed under countable intersections, this property is equivalent to $S_{1}\left(\mathcal{B}_{\Gamma}, \mathcal{B}_{\Gamma}\right)$ asserting that for every sequence $\left\langle\mathcal{U}_{n}: n \in \omega\right\rangle$ of Borel $\gamma$-covers of $X$ there exists $\left\langle U_{n} \in \mathcal{U}_{n}: n \in \omega\right\rangle$ which is a $\gamma$-cover of $X$. Replacing here "Borel" with "clopen" one gets the property $S_{1}\left(\mathcal{C}_{\Gamma}, \mathcal{C}_{\Gamma}\right)$ equivalent to $\alpha_{2}$ of $C_{p}(X)$, see [46]. One of the most intriguing conjectures along these lines is due to Scheepers [48] and says that for subsets of $\mathbb{R}$ the properties $S_{1}\left(\mathcal{C}_{\Gamma}, \mathcal{C}_{\Gamma}\right)$ and $S_{1}(\Gamma, \Gamma)$ coincide, the definition of the latter obtained from that of $S_{1}\left(\mathcal{C}_{\Gamma}, \mathcal{C}_{\Gamma}\right)$ be replacing "clopen" with "open". It follows from the above that for metrizable spaces we have $S_{1}\left(\mathcal{B}_{\Gamma}, \mathcal{B}_{\Gamma}\right)=S_{1}\left(\mathcal{C}_{\Gamma}, \mathcal{C}_{\Gamma}\right)$ in the Laver model, and by

$$
S_{1}\left(\mathcal{B}_{\Gamma}, \mathcal{B}_{\Gamma}\right) \rightarrow S_{1}(\Gamma, \Gamma) \rightarrow S_{1}\left(\mathcal{C}_{\Gamma}, \mathcal{C}_{\Gamma}\right)
$$

the Scheepers' Conjecture is true in the Laver model. However, it is totally unclear what happens under $\mathrm{CH}$. Moreover, if there is $X \subset \mathbb{R}$ satisfying $S_{1}\left(\mathcal{C}_{\Gamma}, \mathcal{C}_{\Gamma}\right)$ but not $S_{1}(\Gamma, \Gamma)$, then this is also obviously true in any forcing extension with the same reals, and hence we can get such a set in a model of CH. Thus if the negative answer to Scheepers' question is consistent, it is also consistent with $\mathrm{CH}$.

Using the key lemma of [29] allowing to analyze names for reals in the Laver model, Miller and Tsaban proved [36] that all $X \subset \mathbb{R}$ satisfying $S_{1}\left(\mathcal{B}_{\Gamma}, \mathcal{B}_{\Gamma}\right)$ must have size $\leq \omega_{1}<\mathfrak{b}=\mathfrak{c}$ in this model, and hence this is also true for $S_{1}\left(\mathcal{C}_{\Gamma}, \mathcal{C}_{\Gamma}\right)$. This answered the question whether in ZFC there exists a set of reals satisfying $S_{1}\left(\mathcal{C}_{\Gamma}, \mathcal{C}_{\Gamma}\right)$ of size $\mathfrak{b}$ in the negative. Their application of Laver's analysis of names of reals found further application to covering properties of products.

One of the basic questions about a topological property is whether it is preserved by finite products. In case of combinatorial covering properties we know that the strongest possible negative result is consistent: Under 
$\mathrm{CH}$ there exist $X, Y \subset \mathbb{R}$ which have the $\gamma$-space property with respect to countable Borel covers2, whose product $X \times Y$ is not Menger, see [37, Theorem 3.2]. Thus the product of spaces with the strongest combinatorial covering property considered thus far might fail to have even the weakest one. This implies that no positive results for combinatorial covering properties can be obtained outright in ZFC. Unlike the vast majority of topological and combinatorial consequences under $\mathrm{CH}$, the latter one does not follow from any equality among cardinal characteristics of the continuum, see [6] and the discussion on [37, p. 2882]. However, there are many other negative results stating that under certain equality among cardinal characteristics $\left(\right.$ e.g., $\operatorname{cov}(\mathcal{N})=\operatorname{cof}(\mathcal{N}), \mathfrak{b}=\mathfrak{d}$, etc $\left.{ }^{3}\right)$ there are spaces $X, Y \subset \mathbb{R}$ with some combinatorial covering property such that $X \times Y$ is not Menger, see, e.g., [4, 39, 54].

Regarding the positive results, until recently the most unclear situation was with the Hurewicz property and the weaker ones. There are two reasons why a product of Hurewicz spaces $X, Y$ can fail to be Hurewicz. In the first place, $X \times Y$ may simply fail to be a Lindelöf space, i.e., it might have an open cover $\mathcal{U}$ without countable subcover. This may indeed happen: in ZFC there are two normal spaces $X, Y$ with a covering property much stronger than the Hurewicz one such that $X \times Y$ does not have the Lindelöf property, see [55, Section 3]. However, the above situation becomes impossible if we restrict our attention to metrizable spaces. This second case turned out to be sensitive to the ambient set-theoretic universe: under $\mathrm{CH}$ there exists a Hurewicz space whose square is not Menger, see [26, Theorem 2.12]. The above result has been achieved by a transfinite construction of length $\omega_{1}$, using the combinatorics of the ideal of measure zero subsets of reals. This combinatorics turned out [54, Theorem 43] to require much weaker set-theoretic assumptions than $\mathrm{CH}$. In particular, under the Martin Axiom there are Hurewicz subspaces of the irrationals whose product is not Menger.

The following theorem, which is the main result which we are going to prove in this article, shows that an additional assumption in the results from [26, 54] mentioned above is really needed.

Theorem 1.1. In the Laver model for the consistency of the Borel's conjecture, the Hurewicz property is preserved by finite products of metrizable spaces. Consequently, the product of any two Hurewicz spaces is Hurewicz if it is Lindelöf.

Let us recall that for metrizable spaces, the separability is equivalent to being Lindelöf, see, e.g., [19, Theorem 4.1.15].

This theorem is a further improvement of the main result of 40 which states that product of two Hurewicz subspaces of $\mathbb{R}$ is Menger in the Laver

\footnotetext{
${ }^{2}$ This property is stronger than $S_{1}\left(\mathcal{B}_{\Gamma}, \mathcal{B}_{\Gamma}\right)$ and we refer the reader to 37] for its definition.

${ }^{3}$ We refer the reader to [10] for the definitions and basic properties of cardinal characteristics of the continuum which are mentioned but are not used in the proofs in this article.
} 
model. The conclusion of Theorem 1.1 does not follow from Borel's conjecture: If we add $\omega_{2}$ many random reals over the Laver model then Borel's conjecture still holds by [7, Section 8.3.B] and we have $\operatorname{cov}(\mathcal{N})=\operatorname{cof}(\mathcal{N})$, and hence in this model there exists a Hurewicz set of reals whose square is not Menger, see [54]. Thus Borel's conjecture is consistent with the existence of a Hurewicz set of reals with even non-Menger square.

Theorem 1.1 seems to be an instance of a more general phenomena, namely that proper posets with fusion affect the behavior of combinatorial covering properties. This happens because sets of reals with certain combinatorial covering properties are forced to have a rather clear structure, which suffices to prove positive preservation results. For instance, the core of the proof of Theorem 1.1 is that Hurewicz subspaces of the real line are concentrated in a sense around their "simpler" subspaces.

Later we shall discuss similar behavior of combinatorial covering properties in the Miller and Sacks models. Now, however, we would like to present a "side effect" of the proof of Theorem 1.1 for maximal almost disjoint families of subsets of $\omega$. Recall that an infinite $\mathcal{A} \subset[\omega]^{\omega}$ is called a mad family, if $\left|A_{0} \cap A_{1}\right|<\omega$ for any distinct $A_{0}, A_{1} \in \mathcal{A}$, and for every $B \in[\omega]$ there exists $A \in \mathcal{A}$ such that $|B \cap A|=\omega$. In [15, Theorem 2.1] Brendle constructed under $\mathrm{CH}$ a mad family $\mathcal{A}$ on $\omega$ such that the Mathias forcing $M_{\mathcal{F}(\mathcal{A})}$ associated to the filter

$$
\mathcal{F}(\mathcal{A})=\left\{F \subset \omega: \exists \mathcal{A}^{\prime} \in[\mathcal{A}]^{<\omega}\left(\omega \backslash \cup \mathcal{A}^{\prime} \subset^{*} F\right)\right\}
$$

adds a dominating real. In the same paper Brendle asked whether such a mad family can be constructed outright in ZFC. This question has been answered in the affirmative in [22] and later independently also in [17] using different methods. However, the mad families constructed there had topological copies of the Cantor set inside, and hence by a simple absoluteness argument are destroyed (i.e., are not maximal any more) by any forcing adding new reals. One can refine Brendle's question in the following way: Suppose that a mad family $\mathcal{A}$ cannot be destroyed by some very "mild" forcing $\mathbb{P}$, i.e., it remains maximal in $V^{\mathbb{P}}$, must then $M_{\mathcal{F}(\mathcal{A})}$ add dominating reals? This refinement makes sense in cases when one is interested in destroying mad families without adding dominating reals and takes some $\mathbb{P}$ which does not add them. Indeed, if $\mathcal{A}$ is already destroyed by $\mathbb{P}$, there is no need to use its Mathias forcing for its destruction in a hypothetic construction of a model where, e.g., $\mathfrak{b}$ should stay small. In what follows we concentrate on the case $\mathbb{P}$ being the Cohen forcing $\mathbb{C}$. Mad families $\mathcal{A}$ which remain maximal in $V^{\mathbb{C}}$ will be called Cohen-indestructible. The following theorem has been proved in [3]

Theorem 1.2. $\mathfrak{p}=\operatorname{cov}(\mathcal{N})=\mathfrak{c}$ implies the existence of a Cohen-indestructible mad family $\mathcal{A}$ such that $M_{\mathcal{F}(\mathcal{A})}$ adds a dominating real.

Recall from [27] that a mad family $\mathcal{A}$ is called $\omega$-mad if for every sequence $\left\langle X_{n}: n \in \omega\right\rangle$ of elements of $\mathcal{F}(\mathcal{A})^{+}$there exists $A \in \mathcal{A}$ such that $\mid A \cap$

\footnotetext{
${ }^{4}$ Since we shall not analyze this poset directly but rather use certain topological characterizations, we refer the reader to, e.g., [15] for its definition.
} 
$X_{n} \mid=\omega$ for all $n$. Cohen-indestructible mad families are closely related to $\omega$-mad ones, see [30] or [27, Theorem 4]: Every $\omega$-mad family is Cohenindestructible, and if $\mathcal{A}$ is Cohen-indestructible, then for every $X \in \mathcal{F}(\mathcal{A})^{+}$ there exists $Y \subset X, Y \in \mathcal{F}(\mathcal{A})^{+}$, such that $\mathcal{A} \uparrow Y=\{A \cap Y: A \in \mathcal{A}, A \cap Y$ is infinite $\}$ is $\omega$-mad as a mad family on $Y$.

The proof of Theorem 1.2 actually gives an $\omega$-mad family. The next theorem shows that $\mathfrak{b}=\mathfrak{c}$ would not suffice in Theorem 1.2 in a strong sense.

Theorem 1.3. In the Laver model for the consistency of the Borel conjecture, for every $\omega$-mad family $\mathcal{A}$ the poset $M_{\mathcal{F}(\mathcal{A})}$ preserves all ground model unbounded sets. In particular, if $\mathcal{A}$ is Cohen-indestructible, then there exists $X \in \mathcal{F}(\mathcal{A})^{+}$such that $M_{\mathcal{F}(\mathcal{A}) \mid X}$ preserves all ground model unbounded sets, where $\mathcal{F}(\mathcal{A})\lceil X$ denotes the filter on $\omega$ generated by the centered family $\{F \cap X: F \in \mathcal{F}(\mathcal{A})\}$.

Theorem 1.3 improves our earlier result proved in 3 asserting, under the same premises, that $M_{\mathcal{F}(\mathcal{A}) \mid X}$ keeps the ground model unbounded. In our proof of Theorem 1.3 we shall not work with the Mathias forcing directly, but rather use the following characterization obtained in 17]: For a filter $\mathcal{F}$ on $\omega$ the poset $M_{\mathcal{F}}$ adds no dominating reals (resp. preserves all ground model unbounded sets) iff $\mathcal{F}$ has the Menger (resp. Hurewicz) covering property when considered with the topology inherited from $\mathcal{P}(\omega)$, which is identified with the Cantor space $2^{\omega}$ via characteristic functions. Thus Theorem 1.3 says that if $A$ is an $\omega$-mad family, then $\mathcal{F}(\mathcal{A})$ is Hurewicz, whereas its predecessor from [3] gave only the Menger property. Let us note that there are ZFC examples of Menger non-Hurewicz filters on $\omega$, see [42]. Theorem 1.3 also intuitively explains why the open question whether there exists an $\omega$-mad family outright in ZFC (it is known and rather easy that they exist under $\mathfrak{b}=\mathfrak{c}$, e.g., in the Laver model) is so difficult: Any such ZFC example should "often" give a mad family whose dual filter is Hurewicz, and it is even unknown how to get one with the Menger filter in ZFC, see [22]. This motivates the following

Question 1.4. Is there a ZFC example of a mad family $A$ such that $\mathcal{F}(\mathcal{A})$ is Hurewicz?

If the answer to this problem is affirmative, then it should probably require some new ideas since with the existing methods it is unclear even how to get a Menger one. On the other hand, a negative answer might be surprisingly easy.

Superficially the conditions in the Miller forcing introduced in 34] are very similar to those in the Laver one, the only difference being that the splitting does not have to occur so often. However, these posets have completely different affects on the behavior of selection principles discussed above. In this context the Miller model has been first investigated in [59], where it has been proved that for sets of reals, the Rothberger property implies the Hurewicz one in this model. This is of course also true in the Laver model because the Borel's conjecture holds there. On the contrary, 
in the Miller model there are uncountable Rothberger spaces (see [38] for a much more stronger result and references therein), so here this implication holds for different, qualitative (and not cardinality) reasons. Let us note that, e.g., under $\mathrm{CH}$ there are Rothberger subspaces of $\mathbb{R}$ which are not Hurewicz, e.g., Luzin sets, see [47.

Another result proved in [59] is that every Menger space in the Miller model is Scheepers, where the latter property is defined in the same way as the Menger one, with the following additional requirement: The selected sequence $\left\{\cup \mathcal{V}_{n}: n \in \omega\right\}$ should be an $\omega$-cover of $X$, which by the definition means that for every finite $F \subset X$ there exists $n$ such that $F \subset \cup \mathcal{V}_{n}$. It is rather straightforward to check that if all finite powers of $X$ have the Menger property, then $X$ is Scheepers. Thus the Miller model was a natural place to address the question whether the Menger property is preserved by finite products of metrizable spaces. The following theorem is the main result of [60].

Theorem 1.5. In the Miller model, the product of any two Menger spaces is Menger provided that it is Lindelöf. In particular, in this model the product of any two Menger metrizable spaces is Menger.

Of course Theorem 1.5 is not true in ZFC as there are equalities between cardinal characteristics which yield even Hurewicz sets of reals with non-Menger product, see our discussion of products of Hurewicz spaces above. Surprisingly, there are also inequalities between cardinal characteristics which imply that the Menger property is not productive even for sets of reals, see [52]. Let us also note that in the Laver model (more generally, under $\mathfrak{b}=\mathfrak{d}$ ) the Menger property is not preserved by products of metrizable spaces, see [39] and [53] for more recent results along these lines.

As we can see in [60], a big part of the proof of Theorem 1.1 (as well as all the results from [59] mentioned above) requires only the inequality $\mathfrak{u}<\mathfrak{g}$ which holds in the Miller model. However, we do not know the answer to the following

Question 1.6. Is the Menger property preserved by finite products of metrizable spaces under $\mathfrak{u}<\mathfrak{g}$ ? If yes, can $\mathfrak{u}<\mathfrak{g}$ be weakened to the Filter Dichotomy or NCF?

We refer the reader to [10, $\S 9]$ for corresponding definitions. Let us note that it is not enough to assume $\mathfrak{u}<\mathfrak{d}$ in Theorem 1.5 as witnessed by the model constructed in [12], see [53]. Natural places to look for a possible negative answer to Question 1.6] are models constructed in [11, 32, 33. Better understanding of these models and possible simplification of the methods used there would be at least as important as a solution of Question 1.6.

What about the products of Hurewicz spaces in the Miller model? The product of finitely many Hurewicz subspaces of $2^{\omega}$ is Menger by Theorem 1.5, and thus the Miller model seemed for a while to be the best candidate for a model where the Hurewicz property is preserved by finite products 
of metrizable spaces. The next theorem proved in [41] refutes this expectation.

Theorem 1.7. In the Miller model there are two $\gamma$-subspaces $X, Y$ of $2^{\omega}$ such that $X \times Y$ is not Hurewicz. In particular, in this model the Hurewicz property is not preserved by finite products of metrizable spaces.

$\gamma$-Spaces were introduced in [21] where it was proved that a Tychonoff space $X$ is a $\gamma$-space if and only if $C_{p}(X)$ has the Frechet-Urysohn property, i.e., for every $f \in C_{p}(X)$ and $A \subset C_{p}(X)$ with $f \in \bar{A}$ there exists $\left\langle f_{n}: n \in\right.$ $\omega\rangle \in A^{\omega}$ converging to $f$. It is well-known that $\gamma$-spaces have the Hurewicz property in all finite powers, see, e.g., [26, Th. 3.6 and Fig. 2] and references therein.

The proof of Theorem 1.7 is based on the fact that if $X \subset 2^{\omega}, X \in V$, and $X$ is a $\gamma$-space in $V$, then $X$ remains a $\gamma$-space in the forcing extension by an iteration of the Miller forcing with countable supports. Previously this was only known for Cohen and random forcing, see [37] and [49], respectively. Let us note that Cohen forcing fails to preserve Hurewicz spaces, see the discussion in [37] after Problem 4.1 therein. This motivates the following

Question 1.8. Does Miller forcing (resp. countable support iterations thereof) preserve the Hurewicz property of ground model spaces which do not contain topological copies of $2^{\omega}$ ? What about (Sierpiński) subspaces of $2^{\omega}$, provided that they exist in the ground model5?

Let us note that $\left(2^{\omega}\right)^{V}$ is not Hurewicz in the Miller model. This can be shown in the same way as that $\left(2^{\omega}\right)^{V}$ is not Menger in the Sacks model. The latter fact is proved in [6] and is attributed there to A. Miller.

Unlike the Laver and Miller models, in the Sacks model introduced in [45] we have the best possible non-preservation by products results: Countable support iterations of the Sacks forcing preserve $\gamma$-subspaces of $\mathbb{R}$ [41], while being non-Menger is obviously preserved by any forcing which does not add unbounded reals. Thus in the Sacks model there exist $\gamma$-spaces $X, Y \subset 2^{\omega}$ with non-Menger product.

The new feature of the Sacks model is that there are rather few Menger sets of reals in this model by the following result obtained in [20].

Theorem 1.9. There are $\mathfrak{c}=\omega_{2}$ many Menger subspaces of $2^{\omega}$ in the Sacks model.

Let us note that by the methods developed in [57] there are at least $\mathfrak{c}^{\mathfrak{d}}$ (resp. $\mathfrak{c}^{\mathfrak{b}}$ ) many Menger (resp. Hurewicz) subspaces of $2^{\omega}$, and hence Theorem 1.9 fails in any model of $\mathfrak{c}=\mathfrak{d}$ including the Laver and Miller one. However, its analogue for Hurewicz spaces might still be true in the Miller model as $\mathfrak{c}^{\mathfrak{b}}=\omega_{2}^{\omega_{1}}=\omega_{2}$ there. This motivates the following

\footnotetext{
${ }^{5}$ Recall that an uncountable $S \subset 2^{\omega}$ is called a Sierpinsski subspace if $|S \cap Z| \leq \omega$ for every $Z$ of Lebesgue measure 0. It is known [26, Theorem 2.10] that Sierpiński subspaces are Hurewicz.
} 
Question 1.10. How many Hurewicz spaces $X \subset 2^{\omega}$ are there in the Miller model? How many concentrated subsets of $2^{\omega}$ are there in the Miller model? In particular, is there a concentrated subset $X$ of $2^{\omega}$ of size $\omega_{2}$ in the Miller model? If not, must every Hurewicz subspace of $2^{\omega}$ of size $\omega_{2}$ contain a topological copy of $2^{\omega}$ ?

A subset $X \subset 2^{\omega}$ is called concentrated on a countable $Q \subset 2^{\omega}$ if $|X \backslash U| \leq$ $\omega$ for any open $U \supset Q$. It is an easy exercise that if $X$ is concentrated on $Q \subset$ $X$ then $X$ is Rothberger, and hence in the Miller model concentrated sets are Hurewicz [59], which naturally relates the different parts of Question 1.10.

\section{Proofs of Theorems 1.1 and 1.3 .}

We shall first introduce several notions crucial for the proof of Theorem 1.1. The first two items of the following definition are due to A. Medini. The third one is an ad hoc notion suitable to our needs.

Definition 2.1. $\quad-X \subset 2^{\omega}$ is called countably controlled if for every $Q \in[X]^{\omega}$ there exists a $G_{\delta^{-}}$-subset $R \supset Q$ of $2^{\omega}$ such that $R \subset X$;

- $X \subset 2^{\omega}$ is called $\sigma$-compactly controlled if for every $\sigma$-compact $Q \subset$ $X$ there exists a $G_{\delta^{-}}$subset $R \supset Q$ of $2^{\omega}$ such that $R \subset X$;

- $X \subset 2^{\omega}$ satisfies property (†) if for every function $R$ assigning to each countable subset $Q$ of $X$ a $G_{\delta^{-}}$subset $R(Q) \supset Q$ of $2^{\omega}$, there exists a family $\mathrm{Q} \subset[X]^{\omega}$ of size $|\mathrm{Q}|=\omega_{1}$ and a mapping $K: \mathrm{Q} \rightarrow \mathcal{P}\left(2^{\omega}\right)$ assigning to every $Q \in \mathrm{Q}$ a $\sigma$-compact subset $K(Q)$ of $R(Q)$, such that $X \subset \cup_{Q \in Q} K(Q)$.

Let us note that under $\mathrm{CH}$ any $X \subset 2^{\omega}$ satisfies $(\dagger)$.

As suggested by Definition 2.1 in what follows we shall work a lot with $G_{\delta}$ subsets of $2^{\omega}$ containing certain countable sets, and hence we need to introduce some auxiliary notation. Given a countable sequence $\left\langle Q_{n}: n \in \omega\right\rangle$ of finite non-empty subsets of $2^{\omega}$ and $u \in \omega^{\uparrow \omega}$, we set

$$
R\left(\left\langle Q_{n}: n \in \omega\right\rangle, u\right):=\left\{x \in 2^{\omega}: \exists^{\infty} n \in \omega \exists q \in Q_{n}(|x-q|<1 / u(n))\right\},
$$

where $\exists^{\infty}$ stands for "exists infinitely many" and we identify $2^{\omega}$ with the standard Cantor subset of $[0,1]$ whenever we use expressions of the form $|a-b|$. As usually, for $x, y \in \omega^{\omega}$ notation $x \leq^{*} y$ means $\{n: x(n)>y(n)\}$ is finite.

Observation 2.2. Let $Q=\bigcup_{n \in \omega} Q_{n} \subset 2^{\omega}$ be a countable union of finite non-empty sets and $R \supset Q$ be a $G_{\delta}$-set. Then there exists $u \in \omega^{\uparrow \omega}$ such that $R\left(\left\langle Q_{n}: n \in \omega\right\rangle, u\right) \subset R$

Proof. Let $R=\bigcap_{n \in \omega} O_{m}$, where each $O_{m}$ is open. For every $m$ we can find $u_{m} \in \omega^{\uparrow \omega}$ such that for every $n$ and $x \in 2^{\omega}$, if there exists $q \in Q_{n}$ such that $|q-x|<1 / u_{m}(n)$, then $x \in O_{m}$. Now any $u \in \omega^{\uparrow \omega}$ such that $u_{m} \leq^{*} u$ for all $m$, is as required.

The following lemma is the key part of the proof of Theorem 1.1. Its proof is reminiscent of that of [36, Theorem 3.2]. We will use the notation from [29] with only differences being that smaller conditions in a forcing 
poset are supposed to carry more information about the generic filter, and the ground model is denoted by $V$. We shall work in $V\left[G_{\omega_{2}}\right]$, where $G_{\omega_{2}}$ is $\mathbb{P}_{\omega_{2}}$-generic and $\mathbb{P}_{\omega_{2}}$ is the iteration of length $\omega_{2}$ with countable supports of the Laver forcing, see [29] for details. As usually, we shall denote $G_{\omega_{2}} \cap \mathbb{P}_{\alpha}$ by $G_{\alpha}$, where $\alpha \leq \omega_{2}$. Following [29], for a condition $p \in \mathbb{P}_{\omega_{2}}$ we denote by $p(0)\langle 0\rangle$ the root of the condition $p(0)$, the latter being the initial coordinate of $p$.

A subset $C$ of $\omega_{2}$ is called an $\omega_{1}-c l u b$ if it is unbounded and for every $\alpha \in \omega_{2}$ of cofinality $\omega_{1}$, if $C \cap \alpha$ is cofinal in $\alpha$ then $\alpha \in C$.

Lemma 2.3. In the Laver model, if $X \subset 2^{\omega}$ has countably-controlled complement $2^{\omega} \backslash X$, then $X$ satisfies $(\dagger)$.

Proof. Let $R$ be such as in the definition of $(\dagger)$. By a standard closing-off argument there exists an $\omega_{1}$-club $C \subset \omega_{2}$ such that for every $\alpha \in C$ the following conditions are satisfied:

- $X \cap V\left[G_{\alpha}\right] \in V\left[G_{\alpha}\right]$;

- For every $Q \in[X]^{\omega} \cap V\left[G_{\alpha}\right]$ the set $R(Q)$ is coded in $V\left[G_{\alpha}\right]$; and

- For every $Q \in\left[2^{\omega}\right]^{\omega} \cap V\left[G_{\alpha}\right]$ disjoint from $X$ there exists a $G_{\delta}$ set $O \supset Q$ coded in $V\left[G_{\alpha}\right]$ such that $O \cap X=\emptyset$.

Let us fix $\alpha \in C$. We claim that $X \subset \bigcup_{Q \in Q} W_{Q}$, where $\mathrm{Q}=[X]^{\omega} \cap V\left[G_{\alpha}\right]$ and $W_{Q}=\bigcup\left\{K: K\right.$ is a compact subset of $R(Q)$ coded in $\left.V\left[G_{\alpha+1}\right]\right\}$. This would complete our proof since it is easy to see (and well-known) that for every $G_{\delta}$ subset $R$ of $2^{\omega}$ and a family $\mathcal{W}$ of fewer than $\mathfrak{b}$ many compact subspaces of $R$, there exists a $\sigma$-compact subspace $K$ of $R$ containing $\bigcup \mathcal{W}$. Note that $\mathfrak{b}=\omega_{2}$ in $V\left[G_{\omega_{2}}\right]$ and there are $\omega_{1}$ many compact sets coded in $V\left[G_{\alpha+1}\right]$.

Suppose that, contrary to our claim, there exists $p \in G_{\omega_{2}}$ and a $\mathbb{P}_{\omega_{2}}$-name $\dot{x}$ such that $p$ forces $2^{\omega} \backslash \dot{X}$ to be countably-controlled and $\dot{x} \in \dot{X} \backslash \bigcup_{Q \in Q} W_{Q}$. By [29, Lemma 11] there is no loss of generality in assuming that $\alpha=0$. Applying [29, Lemma 14] to a sequence $\left\langle\dot{a}_{i}: i \in \omega\right\rangle$ such that $\dot{a}_{i}=\dot{x}$ for all $i \in \omega$, we get a condition $p^{\prime} \leq p$ such that $p^{\prime}(0) \leq 0 p(0)$, and a finite set $U_{s}$ of reals for every $s \in p^{\prime}(0)$ with $p^{\prime}(0)\langle 0\rangle \leq s$, such that for each $\varepsilon>0, s \in p^{\prime}(0)$ with $p^{\prime}(0)\langle 0\rangle \leq s$, and for all but finitely many immediate successors $t$ of $s$ in $p^{\prime}(0)$ we have

$$
p^{\prime}(0)_{t} \wedge p^{\prime} \uparrow\left[1, \omega_{2}\right) \Vdash \exists u \in U_{s}(|\dot{x}-u|<\varepsilon) .
$$

Without loss of generality we may assume that $U_{s} \backslash X \neq \emptyset$ for all $s \in$ $p^{\prime}(0), p^{\prime}(0)\langle 0\rangle \leq s$. Since $2^{\omega} \backslash X$ is countably-controlled, there exists an enumeration $\left\langle s_{n}: n \in \omega\right\rangle$ of $S^{\prime}:=\left\{s \in p^{\prime}(0): p^{\prime}(0)\langle 0\rangle \leq s\right\}$ and $\phi \in \omega^{\uparrow \omega} \cap V$ such that $R^{\prime}:=R\left(\left\langle U_{s_{n}} \backslash X: n \in \omega\right\rangle, \phi\right)$ is disjoint from $X$. Fix a $\mathbb{P}_{\omega_{2}}$-name $\dot{N}$ for a natural number such that $p^{\prime}$ forces that $|\dot{x}-u| \geq 1 / \phi(n)$ for all $n \geq \dot{N}$ and $u \in U_{s_{n}} \backslash \dot{X}$. Let $p^{\prime \prime} \leq p^{\prime}$ be such that $p^{\prime \prime} \uparrow\left[1, \omega_{2}\right)=p^{\prime} \uparrow\left[1, \omega_{2}\right)$ and $p^{\prime \prime}(0)$ is obtained from $p^{\prime}(0)$ by throwing away for every $s_{n} \in S^{\prime}$ finitely many of its successors (as well as all their extensions in $p^{\prime}(0)$ ) so that

$$
p^{\prime \prime}(0)_{s_{n}}{ }^{\wedge} p^{\prime \prime}\left\lceil\left[1, \omega_{2}\right) \Vdash \exists u \in U_{s_{n}}(|\dot{x}-u|<1 /(\phi(n)+1))\right.
$$


for all $n$ such that $s_{n} \in S^{\prime \prime}:=\left\{s \in p^{\prime \prime}(0): p^{\prime \prime}(0)\langle 0\rangle \leq s\right\}$. Replacing $p^{\prime \prime}$ with a yet stronger condition, if necessary, we may additionally assume that $p^{\prime \prime}$ decides $\dot{N}$ as some $N \in \omega$, and $s_{n} \in S^{\prime \prime}$ implies $n \geq N$. Thus we have that

$$
p^{\prime \prime} \Vdash \forall u \in U_{s_{n}} \backslash X(|\dot{x}-u| \geq 1 / \phi(n))
$$

for all $n$ such that $s_{n} \in S^{\prime \prime}$. Combining Equations (11),(2), and (3) we get that for each $\varepsilon>0, s \in S^{\prime \prime}$, and for all but finitely many immediate successors $t$ of $s$ in $p^{\prime \prime}(0)$ we have

$$
p^{\prime \prime}(0)_{t} \wedge p^{\prime \prime} \uparrow\left[1, \omega_{2}\right) \Vdash \exists u \in U_{s} \cap X(|\dot{x}-u|<\varepsilon) .
$$

Fix an enumeration $\left\langle t_{n}: n \in \omega\right\rangle \in V$ of $S^{\prime \prime}$ and find $\psi \in \omega^{\uparrow \omega} \cap V$ such that $R\left(\left\langle U_{t_{n}} \cap X: n \in \omega\right\rangle, \psi\right) \subset R\left(\bigcup_{n \in \omega} U_{t_{n}} \cap X\right)$. Replacing $p^{\prime \prime}$ with a stronger condition $p^{(3)}$ obtained by throwing away for every $t_{n} \in S^{\prime \prime}$ finitely many of its successors (as well as all their extensions in $p^{\prime \prime}(0)$ ), we can get

$$
p_{t_{n}}^{(3) \wedge} p^{(3)} \uparrow\left[1, \omega_{2}\right) \Vdash \exists u \in U_{t_{n}} \cap X(|\dot{x}-u|<1 / 2 \psi(n))
$$

for all $t_{n} \geq p^{(3)}(0)\langle 0\rangle, t_{n} \in p^{(3)}(0)$ (the set of such $t_{n}$ will be denoted by $\left.S^{(3)}\right)$. Let $l$ be the first Laver generic, i.e., $l=\bigcap G_{1} \subset \omega^{<\omega}$. Equation (5) implies that $p^{(3)}$ forces

$$
\dot{x} \in K:=\bigcap_{t_{n} \in l \cap S^{(3)}}\left\{z \in 2^{\omega}: \exists u \in U_{t_{n}}(|z-u| \leq 1 / 2 \psi(n))\right\},
$$

and $K$ is obviously a compact subset of $R\left(\left\langle U_{t_{n}} \cap X: n \in \omega\right\rangle, \psi\right) \subset$ $R\left(\bigcup_{n \in \omega} U_{t_{n}} \cap X\right)$ coded in $V\left[G_{1}\right]$. This contradicts our assumption on $p$ and thus finishes our proof.

The next lemma has been proved in [40]. We present its proof for the sake of completeness.

Lemma 2.4. Let $Y \subset 2^{\omega}$ be Hurewicz and $Q \subset 2^{\omega}$ countable. Then for every $G_{\delta}$-subset $O$ of $\left(2^{\omega}\right)^{2}$ containing $Q \times Y$ there exists a $G_{\delta^{-}}$-subset $R \supset Q$ such that $R \times Y \subset O$.

Proof. Without loss of generality we shall assume that $O$ is open. Let us write $Q$ in the form $\left\{q_{n}: n \in \omega\right\}$ and set $O_{n}=\left\{z \in 2^{\omega}:\left\langle q_{n}, z\right\rangle \in O\right\} \supset Y$. For every $n$ find a cover $\mathcal{U}_{n}$ of $Y$ consisting of clopen subsets of $2^{\omega}$ contained in $O_{n}$. Let $\left\langle\mathcal{U}_{k}^{\prime}: k \in \omega\right\rangle$ be a sequence of open covers of $Y$ such that each $\mathcal{U}_{n}$ appears in it infinitely often. Applying the Hurewicz property of $Y$ we can find a sequence $\left\langle\mathcal{V}_{k}: k \in \omega\right\rangle$ such that $\mathcal{V}_{k} \in\left[\mathcal{U}_{k}^{\prime}\right]^{<\omega}$ and $Y \subset \bigcup_{k \in \omega} Z_{k}$, where $Z_{k}=\bigcap_{m \geq k} \cup \mathcal{V}_{m}$. Note that each $Z_{k}$ is compact and $Z_{k} \subset O_{n}$ for all $n \in \omega$ (because there exists $m \geq k$ such that $\mathcal{U}_{m}^{\prime}=\mathcal{U}_{n}$, and then $Z_{k} \subset \cup \mathcal{V}_{m} \subset O_{n}$ ). Thus $Q \times Y \subset Q \times\left(\bigcup_{k \in \omega} Z_{k}\right) \subset O$. Since $Z_{k}$ is compact, there exists for every $k$ an open $R_{k} \supset Q$ such that $R_{k} \times Z_{k} \subset O$. Set $R=\bigcap_{k \in \omega} R_{k}$ and note that $R \supset Q$ and $R \times Y \subset R \times \bigcup_{k \in \omega} Z_{k} \subset O$.

The next lemma demonstrates the relation between $(\dagger)$ and products with Hurewicz spaces.

Lemma 2.5. Suppose that $\mathfrak{b}>\omega_{1}$. Let $Y \subset 2^{\omega}$ be a Hurewicz space and $X \subset 2^{\omega}$ satisfy $(\dagger)$. Then $X \times Y$ is Hurewicz. 
Proof. Fix a sequence $\left\langle\mathcal{U}_{n}: n \in \omega\right\rangle$ of covers of $X \times Y$ by clopen subsets of $\left(2^{\omega}\right)^{2}$. Thus $X \times Y \subset \bigcap_{n \in \omega} O_{n}$, where $O_{n}=\cup \mathcal{U}_{n}$. By Lemma 2.4 for every

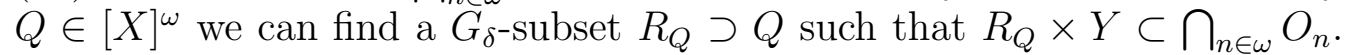
Since $X$ satisfies $(\dagger)$, there exists $Q \subset[X]^{\omega}$ of size $|\mathrm{Q}|=\omega_{1}$, and for every $Q \in \mathrm{Q}$ a $\sigma$-compact $K_{Q} \subset R_{Q}$, such that $K=\bigcup\left\{K_{Q}: Q \in \mathrm{Q}\right\}$ contains $X$ as a subset. It follows that $X \times Y \subset K \times Y \subset \bigcap_{n \in \omega} O_{n}$. It is well-known and easy to see that a product of a compact space and a Hurewicz space is Hurewicz, as well as that the Hurewicz property is preserved by unions of fewer than $\mathfrak{b}$ many subsets of $2^{\omega}$, see [56] and references therein. Thus $K \times Y$ is Hurewicz and $\mathcal{U}_{n}$ is an open cover thereof, therefore for all $n \in \omega$ there exists $\mathcal{V}_{n} \in\left[\mathcal{U}_{n}\right]^{<\omega}$ such that $\left\{\cup \mathcal{V}_{n}: n \in \omega\right\}$ is a $\gamma$-cover of $K \times Y$, and hence it is also a $\gamma$-cover of $X \times Y$ as well.

Finally, we can prove the characterization of Hurewicz subspaces of $2^{\omega}$ which holds in the Laver model and implies Theorem 1.1.

Proposition 2.6. In the Laver model, for a subspace $X$ of $2^{\omega}$ the following conditions are equivalent:

(1) $X$ is Hurewicz;

(2) $X$ satisfies $(\dagger)$;

(3) $2^{\omega} \backslash X$ is countably controlled;

(4) $2^{\omega} \backslash X$ is $\sigma$-compactly controlled; and

(5) $X \times Y$ is Hurewicz for any Hurewicz subspace $Y$ of $2^{\omega}$.

Proof. The equivalence between (1) and (4) is true in ZFC, see [26, Theorem 5.7] or [5, Theorem 1.2]. The implication $(4) \rightarrow(3)$ is obvious outright in ZFC, while $(3) \rightarrow(2)$ is established in Lemma 2.3. The implication $(2) \rightarrow(5)$ is proved in Lemma 2.5 and thus requires only $\mathfrak{b}>\omega_{1}$. And finally, (5) $\rightarrow(1)$ is again obvious in ZFC, take, e.g., $Y$ to be a singleton.

We are now in a position to present the

Proof of Theorem 1.3. By Proposition 2.6 it is enough to show that $\mathcal{F}(\mathcal{A})^{+}$ is countably controlled: This would give that $\mathcal{P}(\omega) \backslash \mathcal{F}(A)^{+}$is Hurewicz, and the map $\mathcal{P}(\omega) \ni Z \mapsto \omega \backslash Z$ is easily seen to be a homeomorphism between $\mathcal{F}(A)$ and $\mathcal{P}(\omega) \backslash \mathcal{F}(A)^{+}$. Let us fix a countable $\mathcal{X} \subset \mathcal{F}(\mathcal{A})^{+}$and find countable infinite $\mathcal{A}_{0} \subset \mathcal{A}$ such that $A \cap X$ is infinite for all $A \in \mathcal{A}_{0}$ and $X \in \mathcal{X}$. It follows that

$$
\left\{Z \in \mathcal{P}(\omega): \forall A \in \mathcal{A}_{0}(|A \cap Z|=\omega)\right\}
$$

is a $G_{\delta}$ subset of $\mathcal{P}(\omega)$ which contains $\mathcal{X}$ and is contained in $\mathcal{F}(\mathcal{A})^{+}$.

By nearly the same argument as at the end of [40] ne can prove that Theorem 1.1 follows from Proposition 2.6. Again, we present its proof for the sake of completeness. A family $\mathcal{F} \subset[\omega]^{\omega}$ is called a semifilter if for every $F \in \mathcal{F}$ and $X \subset \omega$, if $|F \backslash X|<\omega$ then $X \in \mathcal{F}$.

The proof of the first part of Theorem 1.1 uses the characterization of the Hurewicz property obtained in [59]. Let $u=\left\langle U_{n}: n \in \omega\right\rangle$ be a sequence of subsets of a set $X$. For every $x \in X$ let $I_{s}(x, u, X)=\left\{n \in \omega: x \in U_{n}\right\}$. If every $I_{s}(x, u, X)$ is infinite (the collection of all such sequences $u$ will be 
denoted by $\left.\Lambda_{s}(X)\right)$, then we shall denote by $\mathcal{U}_{s}(u, X)$ the smallest semifilter on $\omega$ containing all $I_{s}(x, u, X)$. By [59, Theorem 3], a Lindelöf topological space $X$ is Hurewicz if and only if for every $u \in \Lambda_{s}(X)$ consisting of open sets, the semifilter $\mathcal{U}_{s}(u, X)$ is Hurewicz. The proof given there also works if we consider only those $\left\langle U_{n}: n \in \omega\right\rangle \in \Lambda_{s}(X)$ such that all $U_{n}$ 's belong to a given base of $X$.

Proof of Theorem 1.1. Suppose that $X, Y$ are Hurewicz spaces such that $X \times Y$ is Lindelöf and fix $w=\left\langle U_{n} \times V_{n}: n \in \omega\right\rangle \in \Lambda_{s}(X \times Y)$ consisting of open sets. Set $u=\left\langle U_{n}: n \in \omega\right\rangle, v=\left\langle V_{n}: n \in \omega\right\rangle$, and note that $u \in \Lambda_{s}(X)$ and $v \in \Lambda_{s}(Y)$. It is easy to see that

$$
\mathcal{U}_{s}(w, X \times Y)=\left\{A \cap B: A \in \mathcal{U}_{s}(u, X), B \in \mathcal{U}_{s}(v, Y)\right\},
$$

and hence $\mathcal{U}_{s}(w, X \times Y)$ is a continuous image of $\mathcal{U}_{s}(u, X) \times \mathcal{U}_{s}(v, Y)$. By [59, Theorem 3] both of latter ones are Hurewicz, considered as subspaces of $2^{\omega}$, and hence their product is a Hurewicz space by Proposition 2.6. Thus $\mathcal{U}_{s}(w, X \times Y)$ is Hurewicz, being a continuous image of a Hurewicz space. It now suffices to use [59, Theorem 3] again.

\section{REFERENCES}

[1] Arkhangel'skiı̌, A., The spectrum of frequencies of a topological space and the product operation, Trudy Moskov. Mat. Obshch. 40 (1979), 171-206.

[2] Arkhangel'skiı̌, A., Topological function spaces. Mathematics and its Applications (Soviet Series), 78. Kluwer Academic Publishers Group, Dordrecht, 1992.

[3] Aurichi, L.; Zdomskyy, L. Covering properties of $\omega$-mad families, submitted.

[4] Babinkostova, L., On some questions about selective separability, Math. Log. Quart. 55 (2009), 539-541.

[5] Banakh, T.; Zdomskyy, L., Separation properties between the $\sigma$-compactness and Hurewicz property, Topology Appl. 156 (2008), 10-15.

[6] Barman, D.; Dow, A., Proper forcing axiom and selective separability, Topology Appl. 159 (2012), 806-813.

[7] Bartoszyński, T.; Judah, H., Set theory. On the structure of the real line. A. K. Peters, Ltd., Wellesley, MA, 1995.

[8] Bartoszynski, T.; Shelah, S., Continuous images of sets of reals, Topology Appl. 116 (2001), 243-253.

[9] Bartoszynski, T.; Tsaban, B., Hereditary topological diagonalizations and the Menger-Hurewicz Conjectures, Proc. Amer. Math. Soc. 134 (2006), 605-615.

[10] Blass, A., Combinatorial cardinal characteristics of the continuum, in: Handbook of Set Theory (M. Foreman, A. Kanamori, and M. Magidor, eds.), Springer, 2010, pp. 395-491.

[11] Blass, A.; Shelah, S., There may be simple $P_{\aleph_{1}}$ - and $P_{\aleph_{2}}$-points and the RudinKeisler ordering may be downward directed, Ann. Pure Appl. Logic 33 (1987), 213-243.

[12] Blass, A.; Shelah, S., Ultrafilters with small generating sets, Israel J. Math. 65 (1989), 259-271.

[13] Borel, E., Sur la classification des ensembles de mesure nulle, Bull. Soc. Math. France 47 (1919), 97-125.

[14] Bukovsky, L., The structure of the real line. Instytut Matematyczny Polskiej Akademii Nauk. Monografie Matematyczne (New Series), 71. Birkhäuser/Springer Basel AG, Basel, 2011. 
[15] Brendle, J., Mob families and mad families, Arch. Math. Logic 37 (1998), 183-197.

[16] Chodounsky, D.; Guzman, O,; Hrušák, M., Mathias-Prikry and Laver type forcing; Summable ideals, coideals, and +-selective filters, Arch. Math. Logic 55 (2016), 493-504.

[17] Chodounský, D.; Repovš, D.; Zdomskyy, L., Mathias forcing and combinatorial covering properties of filters, J. Symb. Log. 80 (2015), 1398-1410.

[18] Dow, A., Two classes of Fréchet-Urysohn spaces, Proc. Amer. Math. Soc. 108 (1990), 241-247.

[19] Engelking, R., General topology. Second edition. Sigma Series in Pure Mathematics, 6. Heldermann Verlag, Berlin, 1989.

[20] Gartside, P.; Medini, A.; Zdomskyy, L., The Vietoris hyperspace $\mathcal{K}(X)$ is hereditarily Baire if and only of $X$ is co-Menger, work in progress.

[21] Gerlits, J., Nagy, Zs., Some properties of $C(X)$, I, Topology Appl. 14 (1982), 151-161.

[22] Guzmán, O.; Hrušák, M.; Martínez, A.A., Canjar filters, Notre Dame J. Formal Logic 58 (2017), 79-95.

[23] Hrušák, M.; van Mill, J., The existence of a meager in itself CDH metric space is independent of ZFC, Proc. Amer. Math. Soc. 146 (2018), 2689-2695.

[24] Hurewicz, W., Über die Verallgemeinerung des Borellschen Theorems, Math. Z. 24 (1925), 401-421.

[25] Hurewicz, W., Über Folgen stetiger Funktionen, Fund. Math. 9 (1927), 193204.

[26] Just, W.; Miller, A.W.; Scheepers, M.; Szeptycki, P.J., The combinatorics of open covers. II, Topology Appl. 73 (1996), 241-266.

[27] Kurilić, M.S., Cohen-stable families of subsets of integers, Journal of Symbolic Logic 66 (2001), 257-270.

[28] Kuratowski, C., Topologie. I et II. (French) [Topology. I and II] Part I with an appendix by A. Mostowski and R. Sikorski. ditions Jacques Gabay, Sceaux, 1992.

[29] Laver, R., On the consistency of Borel's conjecture, Acta Math. 137 (1976), 151-169.

[30] Malykhin, V.I., Topological properties of Cohen generic extensions, Trudy Moskov. Mat. Obshch. 52 (1989), 3-33.

[31] Menger, K., Einige Überdeckungssätze der Punktmengenlehre, Sitzungsberichte. Abt. 2a, Mathematik, Astronomie, Physik, Meteorologie und Mechanik (Wiener Akademie) 133 (1924), 421-444.

[32] Mildenberger, H.; Shelah, S., The principle of near coherence of filters does not imply the filter dichotomy principle, Trans. Amer. Math. Soc. 361 (2009), 2305-2317.

[33] Mildenberger, H., Local Ramsey Spaces in Matet Forcing Extensions and Finitely Many Near-Coherence Classes, submitted.

[34] Miller, A., Rational perfect set forcing, in: Axiomatic Set Theory (J. Baumgartner, D. A. Martin, S. Shelah, eds.), Contemporary Mathematics 31, American Mathematical Society, Providence, Rhode Island, 1984, pp. 143-159.

[35] Miller, A. W., Special subsets of the real line, in: Handbook of Set-Theoretic Topology (K. Kunen, J.E. Vaughan, eds.), North Holland, Amsterdam, 1984, pp. 201-233.

[36] Miller, A.W.; Tsaban, B., Point-cofinite covers in the Laver model, Proc. Amer. Math. Soc. 138 (2010), 3313-3321.

[37] Miller, A.W.; Tsaban, B.; Zdomskyy, L., Selective covering properties of product spaces, II: gamma spaces, Trans. Amer. Math. Soc. 368 (2016), 2865-2889.

[38] Orenshtein, T.; Tsaban, B., Linear $\sigma$-additivity and some applications, Trans. Amer. Math. Soc. 363 (2011), 3621-3637. 
[39] Repovš, D.; Zdomskyy, L., On M-separability of countable spaces and function spaces, Topology Appl. 157 (2010), 2538-2541.

[40] Repovš, D.; Zdomskyy, L., Products of Hurewicz spaces in the Laver model, Bull. Symb. Log. 23 (2017), 324-333.

[41] Repovš, D.; Zdomskyy, L., Preservation of $\gamma$-spaces and covering properties of products, Proc. Amer. Math. Soc. 147 (2019), 4979-4985.

[42] Repovš, D.; Zdomskyy, L., Zhang, S., Countable dense homogeneous filters and the Menger covering property, Fund. Math. 224 (2014), 233-240.

[43] Rothberger, F., Eine Verschärfung der Eigenschaft C, Fund. Math. 30 (1938), 50-55.

[44] Rothberger, F., Sur les familles indenombrables de suites de nombres naturels et les problemes concernant la propriete C, Proc. Cambridge Philos. Soc. 37 (1941), 109-126.

[45] Sacks, G.E., Forcing with perfect closed sets, in: Axiomatic Set Theory (D. Scott eds.), Proc. Symp. in Pure Math. Vol. XIII, Part I. Am. Math. Soc., Providence, RI, 1971, 331-355.

[46] Sakai, M., The sequence selection properties of $C_{p}(X)$, Topology Appl. 154 (2007), 552-560.

[47] Scheepers, M., Combinatorics of open covers. I. Ramsey theory, Topology Appl. 69 (1996), 31-62.

[48] Scheepers, M., Sequential convergence in $C_{p}(X)$ and a covering property, EastWest J. Math. 1 (1999), 207-214.

[49] Scheepers, M., Gerlits and function spaces, Studia Sci. Math. Hungar. 47 (2010), 529-557.

[50] Scheepers, M.; Tall, F., Lindelöf indestructibility, topological games and selection principles, Fund. Math. 210 (2010), 1-46.

[51] Sierpiński, W., Sur un ensemble non dénombrable, dont toute image continue est de mesure nulle, Fund. Math. 11 (1928), 302-303.

[52] Szewczak, P.; Tsaban, B., Products of Menger spaces: a combinatorial approach, Ann. Pure Appl. Logic 168 (2017), 1-18.

[53] Szewczak, P.; Tsaban, B.; Zdomskyy, L., Finite powers and products of Menger sets, submitted.

[54] Scheepers, M.; Tsaban, B., The combinatorics of Borel covers, Topology Appl. 121 (2002), 357-382.

[55] Todorčević, S., Aronszajn orderings. Djuro Kurepa memorial volume, Publ. Inst. Math. (Beograd) (N.S.) 57(71) (1995), 29-46.

[56] Tsaban, B., Additivity numbers of covering properties, in: Selection Principles and Covering Properties in Topology (L. Kovinac, editor), Quaderni di Matematica 18, Seconda Universita di Napoli, Caserta 2006, 245-282.

[57] Tsaban, B., Zdomskyy, L., Scales, fields, and a problem of Hurewicz, J. Eur. Math. Soc. (JEMS) 10 (2008), 837-866.

[58] Tsaban, B.; Zdomskyy, L., Hereditarily Hurewicz spaces and Arhangel'ski sheaf amalgamations, J. Eur. Math. Soc. (JEMS) 14 (2012), 353-372.

[59] Zdomskyy, L., A semifilter approach to selection principles, Comment. Math. Univ. Carolin. 46 (2005), 525-539.

[60] Zdomskyy, L., Products of Menger spaces in the Miller model, Adv. Math. 335 (2018), 170-179.

Kurt Gödel Research Center for Mathematical Logic, University of Vienna, Währinger Strasse 25, A-1090 Wien, Austria.

E-mail address: lzdomsky@gmail.com

$U R L:$ http://www.logic.univie.ac.at/ Izdomsky/ 